\title{
Design of Digital Network Shared Learning Platform based on SCORM Standard
}

\author{
https://doi.org/10.3991/ijet.v13i07.8602 \\ Yan-Hong Shen \\ Zhejiang Industry Polytechnic College, Zhejiang, China \\ yhshen9870@21cn. com
}

\begin{abstract}
Aiming at the problem of low utilization rate of teaching resources, a personalized recommendation system of digital teaching resources under SCORM standard was put forward. Based on the learner's learning process, standards and personalized recommendation techniques, a SCORM digital teaching resource management model was constructed. According to the recommendation algorithm of collaborative filtering in personalized recommendation technology, a digital teaching resource recommendation model based on SCORM standard was constructed. The SCORM digital teaching resource library system based on collaborative filtering technology was designed and implemented. The system was evaluated. The data of the assessment were analyzed. The results showed that the recommendation system had a good promoting effect on the learners. Therefore, the system can provide personalized recommendations for learners. It improves learning efficiency.
\end{abstract}

Keywords-SCORM, collaborative filtering, digital resources

\section{$1 \quad$ Introduction}

With the rapid development of computer science and technology, the popularity of distance education has made us enter the era of E-learning. Because online E-learning teaching has many advantages, such as being free from constraints of time and space, and low learning cost, it has become one of the most successful methods of education in computer-assisted education. The E-learning platform provides a channel for teaching resources for teachers, students and training institutions, so that students can learn at anytime and anywhere. The success of E-learning teaching depends mainly on two aspects: how to provide the best teaching resources with the least cost and how to make the learners achieve a better teaching effect. Therefore, the collaborative filtering recommendation algorithm and the SCORM standard are introduced into the Elearning. It improves the reusability of online teaching resources and promotes the efficiency of online teaching.

With the continuous development of computer technology and communication technology, the speed of knowledge updating is fast. There is an urgent need for a kind of teaching method that can be studied anytime and anywhere. Because of its rich teaching resources (text, audio and video) form, and unified learning platform 
(browser), E-learning is welcomed by learners. Nowadays, both the government and the enterprises begin to use E-learning to train the learners. There are many online training network platforms. However, the teaching resources between each online teaching platform cannot be reused, which has caused a great waste of resources. In 2010, China released the "National Medium and Long-Term Education Reform and Development Plan (2010-2020)". It clearly puts forward "strengthening the development and application of high-quality teaching resources, strengthening the construction of network teaching resources, introducing the international high-quality digital teaching resources, developing online learning courses, and promoting the popularization and sharing of high-quality teaching resources". This shows that the construction and sharing of digital teaching resources has been paid more and more attention. At present, online teaching resources are increasing at a geometric level. When learners face so many teaching resources, they cannot distinguish the advantages and disadvantages of the resources, which leads to a decline in the efficiency of teaching.

In this context, SCORM is the current global unified resource standard. The aim is to ensure that the learners can build a high-quality learning resource. It provides a good standard for implementation and sharing of resources. In addition, collaborative filtering technology, as a more mature personalized recommendation method in ecommerce, can be applied to online teaching resources. It can also solve the problem of learners' "selective difficulty in teaching resources".

\section{State of the art}

\subsection{Overview of SCORM}

The development of teaching resources is an important link in network teaching. After the development of the network platform is completed, a large amount of financial and material resources has been devoted to developing teaching courseware resources. Using the SharePoint learning Suite (SLK), Rimale, Z. et al. [1] designed a new method of response standard design (rscod) based on semantic learning objects (SLO). Silva, P. J. E. D. et al. [2] pointed out that due to the lack of unified development standards, teaching resources cannot be reused. Based on the knowledge ontology and the SCORM specification, Zhu, X. H. et al. [3] established an interoperable model of intelligent content objects. Seixas, D. [4] pointed out that the resources of a platform can only be used under the platform, which greatly improves the development cost of online teaching resources platform. Moreover, because of the lack of information sharing between resources and resources, the flexibility of the whole teaching resource platform is not high. Herbert, C. et al. [5] explored the application patterns of mixed learning in large group teaching. Scientific research institutions have also paid more and more attention to these problems.

Neves, D. E. et al. [6] gave the characteristics of SCORM standard. First, sustainability. Teaching resources will not be invalid because of the update of technology. It can be used for a long time. Second, reusability. Teaching can basically be used without modification. It can be reused in different platforms, and can be combined with 
other teaching contents according to their needs. Third, interoperability. Because teaching materials follow a unified standard, it can be presented on any standard platform, or can be modified by editing tools that conform to the standard. Fourth, availability. With the platform, learners can read the learning and teaching resources through the Internet without any time and space constraints, so as to achieve the purpose of distance learning.

In order to realize the digital teaching resource library that meets the SCORM standard, the platform developer and the textbook designer should understand the components of the SCORM. The SCORM 2004 is composed mainly of three parts: the content aggregation model CAM, the runtime environment RTE, the sort and the navigation SN.

\subsection{Personalized recommendation technology}

In the traditional system platform, the user browsing platform can only receive the information provided by the platform passively. Because the needs of each user are different, it is impossible to serve each user. Personalized recommendation technology enables the system to transform from a passive information provider to "what users need, what we provide", which is more targeted than traditional way. By collecting and analyzing user information, personalized recommendation technology can predict user behavior. It is better to provide the users with their corresponding services, to achieve the purpose of personalized service. At present, personalized recommendation technology can be divided into user-based recommendation, content-based recommendation, collaborative filtering recommendation.

The recommendation advantage based on user statistics is that: First, because it does not depend on the user's behavior information, there is no cold start problem. Second, because it does not depend on the information of the resource itself, it can be used within the various fields. However, the shortcoming of recommendation based on user statistics is also obvious. This recommendation does not analyze resource information and user behavior. When there are many kinds of resources, it is impossible to make a good recommendation.

The content-based recommendation mechanism can model user preference resources well, which is more accurate than the user's statistical recommendation. However, there are several problems in this recommendation. It is necessary to use the user's history of preference for resources, so there is a cold start problem. Ruano, I. et al. [7] pointed out that content-based recommendations only analyze the similarity of resources, it does not consider the user's attitude towards resources. As the recommendation is based on information from resources, the detailed description of the resource determines the quality of the recommendation.

With the rapid development of Web2.0, more and more websites require interaction with users, and collaborative filtering technology emerges as the times require. The principle of collaborative filtering recommendation is to discover the correlation between resources and resources based on users' preferences for goods or resources, or to find correlations among users, and then recommend them based on these relevancies. Collaborative filtering recommendation is mainly divided into user - based 
recommendation and project - based recommendation. Based on collaborative filtering, personalized recommendation mechanism is one of the most widely used methods of recommendation. It has the following two significant advantages. First, because it does not depend on the attributes of the goods, it does not need to strictly model the objects or users, and does not require the description of the items to be machine understandable. Therefore, this method can be applied to some difficult areas, such as movies, music, etc. Second, since the recommendation is the subjective evaluation of the user, it represents the true reflection of the user's interest, so the accuracy of the recommendation is better. In addition, the experience between users is shared. The potential interest of the user was found.

\subsection{Overview of SCORM digital teaching resource management model}

In the research content of the current researchers, there is a lack of a unified standard for teaching resources in the digital teaching resources database. There is also a lack of contact and recommendation between resources and resources. To solve this problem, the problems existing in the current digital teaching resource management are found out first. Based on the two achievements of SCORM standard and personalized recommendation at home and abroad, Lindert [8] established a SCORM digital teaching management model.

In order to solve the personalized problem in the use of the digital teaching system, the SCORM system, the personalized recommendation algorithm and the personalized learning model are analyzed. The digital teaching management model of SCORM standard is put forward from the three time periods of teaching activities in the before middle and post period by Ueda and Nakamura.

In the pre-teaching stage, the learners can choose their favorite courses through the list of teachers defined courses or the recommended list of personalized recommendation systems. Since the recommended course is acquired by learners' subjective evaluation and behavioral data analysis, it is more suitable for learners to learn the next classification after finishing a class.

The stage of teaching activity is the most closely connected stage between the learners and the LMS. Recommendation engine in the system needs to analyze learners' personal information, learning progress, learners' evaluation of a course, and determine suitable courses for learners, and recommend them to learners.

After teaching activities, it includes the assessment of learners. If learners have a good command of a topic, they can enter the next course. If learners do not reach the basic level of mastery, they will need to repeat the course. In addition, after the completion of learning assessment, the system will require learners to evaluate their current learning topics. The score of the system will also affect the recommendation of the recommended curriculum list, to achieve the purpose of personalized recommendation.

Based on the combination of teaching resources and specific organizational structure, SCORM defines the combination of simple learning materials and shared learning resources into complex and reusable learning content objects. The textbook after the SCORM standard is a package exchange file PIF. A package represents a set of 
independent learning contents. It can be not only a part of the course, but also a complete course or a collection of thousands of courses. A package can be reassembled or split. The package must be able to exist independently. It contains all the information needed when it is run or reused alone. PIF is generally a compressed package in the form of storage. Of course, it can also release resources through CD or mobile settings media. The biggest role of content packaging is to establish binding concerns between content objects and metadata. The structure of physical and logical components is separated. A PIF package contains two parts: the part of the physical file and the list of the contents. Physical resources include the learning of content files, various types of media materials, and evaluation documents.

The content list is used to describe the content fabric in the content package. Content packaging should conform to the IMS content package binding specification. It put together and control file placement within the PIF sub root record. The content list is mainly composed of four parts: metadata, organization structure, resource reference, and subcontent list. Metadata is the data that describes the data. Organizational structure is used to describe the organization of learning resources. Resource references include references to media material and actual resources in the list of contents. The resources that are referenced can be Asset and SCO. A subcontent list is a nested list of contents, which is the entrance to the data description of the next content package.

According to the SCORM standard, Ueda, H. and Nakamura, M. [9] pointed out that the teaching resources are encapsulated into a PIF package. It can be uploaded to the SCORM standard teaching resource library. The teaching resource database is used to decompress the XML list file in the package. Then, the learning content information is added to the resource library. The import process is divided into three steps. The SCORM standard ZIP package is uploaded to the server. The ZIP package is decompressed and the imsmanifest.xml file is parsed. The course information in the XML file is parsed and stored in the database.

The SCORM runtime environment provides a common mechanism. SCO can run on LMS. In order to allow LMS to read the related state information of the SCORM, RTE must provide a unified API and data model. SCORM provides the API function at the beginning of LMS and the data model at the beginning of the CMI. Men, W. et al. [10] found that through these functions and data models, the Javascript is called to obtain the related state information of the SCO, thus tracking the state of the learner. SCORM SCO and LMS interaction process is shown in Figure 1.

In the SCORM standard, the state of the learner's behavior is tracked. The data is passed between LMS and SCO. The data model defines the data specification for the data communication between the learning content object and the learning management system. SCO can be identified and tracked in different LMS, and the state information of SCO can be recorded in a unified way. Since the data model comes from AICC CMI, all the names of the data elements begin with "cmi". The RTE data model is shown in Figure 2. 


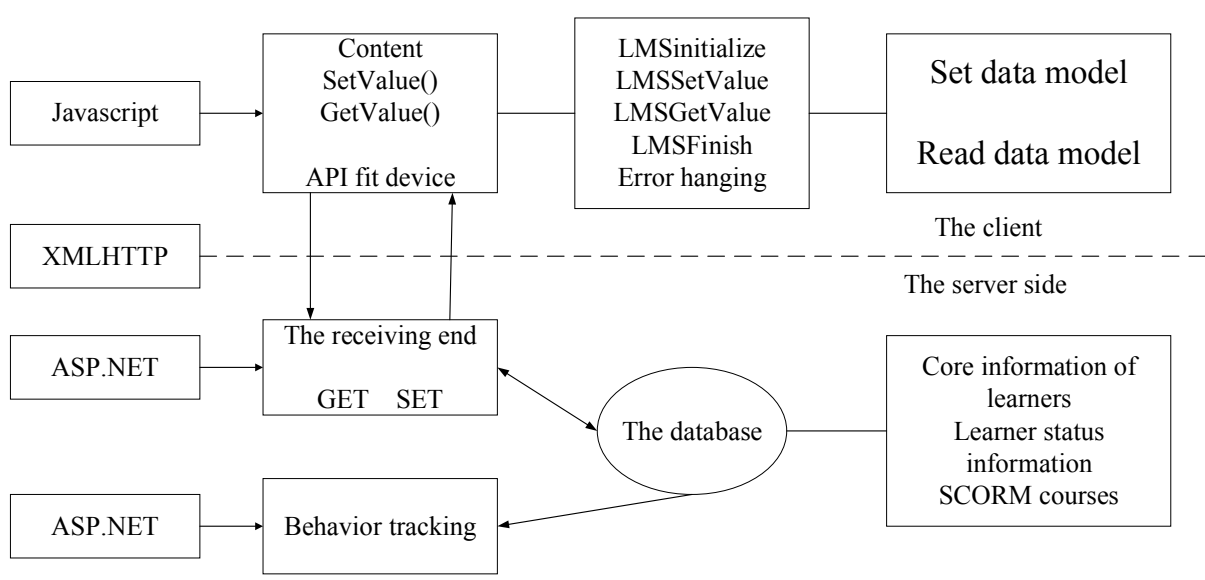

Fig. 1. SCORM SCO and LMS interaction process

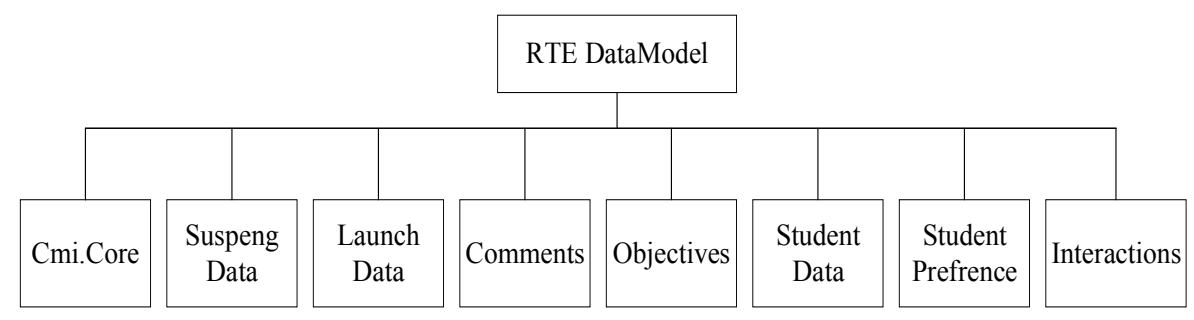

Fig. 2. The RTE data model

\subsection{Collaborative filtering recommendation of digital teaching resources}

According to the characteristics of SCORM learning resources and learners' learning behavior, a collaborative filtering recommendation model of digital teaching resources is proposed. In this model, the behavior and information of the learners are modeled and the model is formed. Then, the filter strategy is selected according to the current system situation, and the influence factors of the learner's behavior are added to form the nearest neighbor set. Multi-media online learning system is recommended to learners. Learner interaction with the system will have an impact on the entire recommendation system. An organic whole is formed. The collaborative filtering recommendation model of digital teaching resources is shown in Figure 3. 


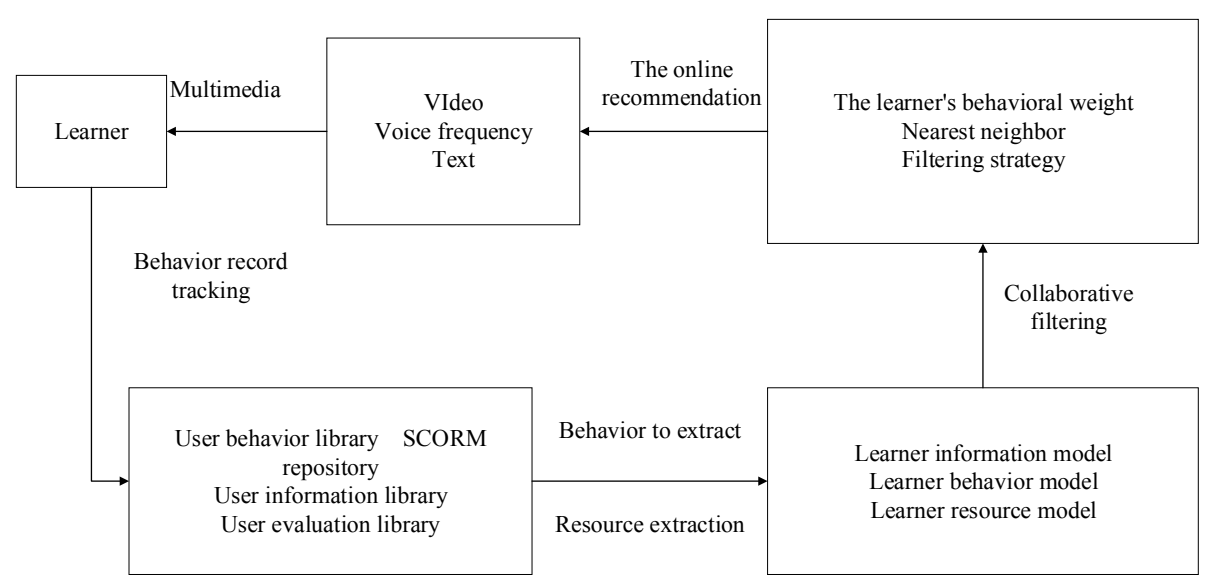

Fig. 3. The collaborative filtering recommendation model of digital teaching resources

The system information base consists of four parts:

User information base: It mainly refers to the user's registration information, such as name, gender, age, education and so on. Through data mining technology, user information is extracted. User information should be very detailed, which will affect the online initial system recommended.

User behavior library: When users learn SCORM resources, they will have learning progress status and learning actions such as resource browsing progress, favorites, downloading and the like. This information is recorded in the user behavior library through a learning tracking mechanism.

SCORM resource library: After designing the classes for various media types (eg, text, audio, video), instructional designers upload the SCO to the SCORM repository, which stores the resource information. Due to the large number of resources in the resource pool, the recommendation system has added social tags to each resource. The introduction of tags helps to classify the resource contents and achieve the uniform management and sharing of resources.

User evaluation library: After learning a particular course, learners rate the level of satisfaction of the course. The evaluation data will be recorded in the user evaluation database. The level of detail of the user review library will affect the accuracy of the collaborative filtering recommendation.

The repository is the source of data for the entire digital collaboration system. In this model, the learner's behavior model is established by collecting and recording the learner's learning behavior and mining the learner's trajectory of learning behavior. The digital model is in the "data converter" role throughout the system. After the system mines the information of the repository, it turns the data into information that can be identified by the collaborative filtering engine. This process is the digital normalization process. After the standardization is completed, the resource information is transformed into a digital model in preparation for personalized recommendation.

Collaborative filtering engine is the core of personalized recommendation system. The engine excavated user information. The "isolated" resources with socialized la- 
bels in the learning resource library are associated. The learner's behavior model is digitalized as the learning weight value. The appropriate recommendation strategy is chosen. The candidate recommendation resource set is generated. In a variety of media presentation, the learner recommends the learning resources that they may be interested in. Collaborative filtering engine can effectively solve the problems of low level of learning resources construction, which is not conducive to the search of learners and so on, so as to ensure the quality of personalized recommendation.

The collaborative filtering algorithms are based on the following assumptions: First, the interest between the users is similar. Second, the user's operational evaluation of resources includes their interest preferences. Therefore, it is used as a basis for predicting projects for other users. The collaborative filtering algorithm is introduced into the field of digital teaching. An improved collaborative filtering algorithm is proposed for the sparsity of matrix. The traditional way to solve the problem of matrix sparsity is to add the default value to the matrix. Although this method can alleviate the sparsity of the matrix to a certain extent, it can't give a correct analysis of the user's interest tendency. Based on the above problem, when the matrix is initialized, if user evaluation is less, users' registration information, learning state information and other behaviors of resource operation such as browsing and collecting are excavated. The user's behavior is added to the user's similarity calculation as a weight value. Compared with the traditional collaborative filtering algorithm, the algorithm can effectively solve the sparse problem of matrix, and the accuracy of recommendation has also been greatly improved. The improved collaborative filtering recommendation process is shown in Figure 4.

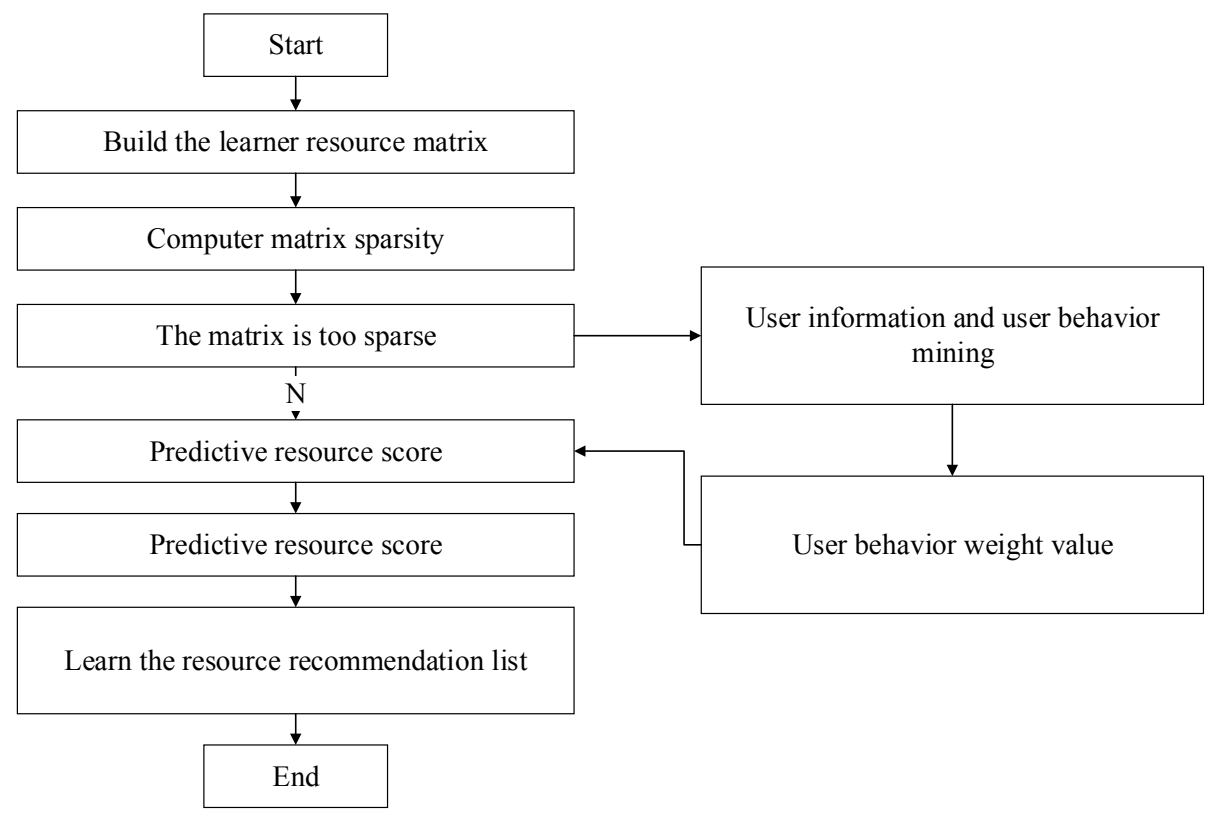

Fig. 4. The improved collaborative filtering recommendation process 
To sum up, in the past research, the cost of network teaching resources is great, and the efficiency of network teaching is low. In addition, it is difficult for learners to choose the teaching resources. In order to improve the utilization rate of teaching resources, a personalized recommendation system of digital teaching resources un-der SCORM standard was put forward. A SCORM digital teaching resource management model was constructed. The SCORM digital teaching resource library system based on collaborative filtering technology was designed and implemented. The system was evaluated. The data of the assessment were analyzed. The proposed method improves learning efficiency.

\section{Methodology}

\subsection{The requirement analysis of the system}

In addition to the previously described personalized recommendation system module, as a whole digital teaching system, it also includes the course upload, editing, learning and other functions. Through analysis, a demand analysis model is proposed, as shown in Figure 5 and Figure 6.

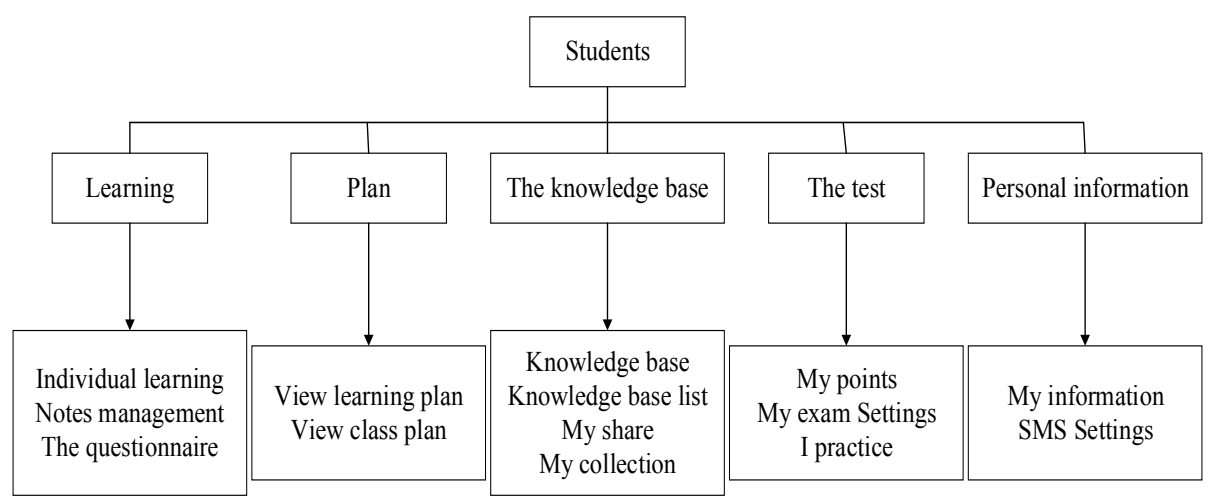

Fig. 5. Use case diagram of digital resource system (a)

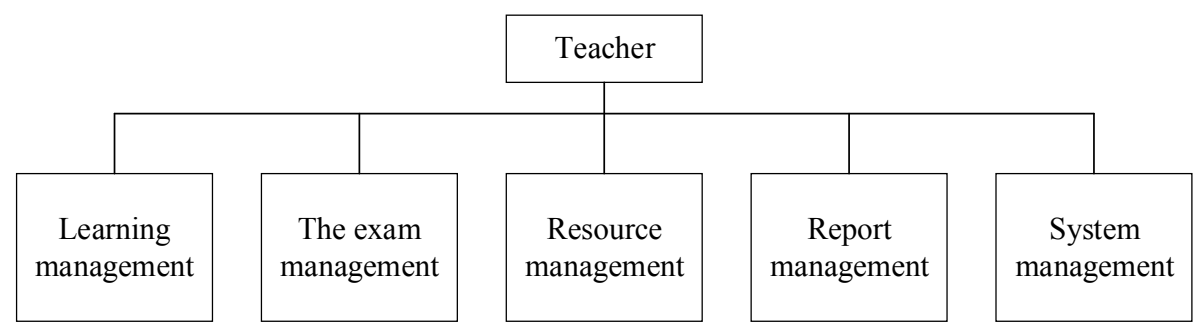

Fig. 6. Use case diagram of digital resource system (b) 


\subsection{Model}

The design of database model is the foundation of the whole system. The stability of the system is based on a good database design.

UserInfo table: user information table. It is used to store the basic information of the learners, such as login username, password, sex, age, educational level, and so on.

LessonInfo: the course information table. Users keep the basic information on the course, such as the title of the course, the content of the course, the payment, the period of study, and so on.

UserLesson: user selection table. It is used to store the information of elective courses for each scholar, such as the beginning of the course, the end time, the completion of the study, and so on.

Content: the course content table. It is used to store the specific content of the course, such as the title of the course, the metadata of the course, the content of the course and so on.

SCORM: a data sheet for the SCORM course. The table is one of the most important tables in the platform. Each field in the table corresponds to the data model in the RTE. The content of the data model will eventually be submitted to the table.

Question: user answer table. It is used to store information about teachers' questions after the completion of the user's learning.

Rule: the table of teaching rules. It is used to store the sequence of course learning in the platform, which shows the design of the instructional designers on the structure of the curriculum. The value of the table can be derived from the teacher's data import.

Exam: a test sheet. After the learners have completed their study, the user tests the learners to determine whether they need to redo the course.

(Lesson/Content/Question) Type table: the user uses it to store information about the course information and the content of the course.

\subsection{System architecture}

Based on collaborative filtering technology, SCORM digital teaching resource library is divided into presentation layer, network layer, functional and logical control layer and data layer from the architecture. The data layer is used to store the corresponding course information and user information of the SCORM course. The function and logic control layer is used to extract data from the data layer. Then, the module of the processing program is worked out. The presentation layer is used to display the data of the function and logic control layer to the user.

\section{$4 \quad$ Result Analysis and Discussion}

The personalized recommendation of the digital teaching resource base is based on the collaborative filtering technology. The score predicted by collaborative filtering algorithm is very important. The similarity between its prediction score and user's 
actual score can represent the recommendation accuracy of the recommendation system, so as to judge the performance of the system.

\subsection{Selection of data sets}

In order to test the algorithm performance of digital teaching resource library, a wide range of data sets Movielens is used to evaluate the algorithm. Movielens is a web site Project at the University of Minnesota. The movie website has a large number of users of their favorite movie evaluation data. The data set of the site is divided into three categories $(100 \mathrm{k}, 1 \mathrm{M}, 10 \mathrm{M})$, and the small data sets selected in this paper are evaluated. The data set contains 100 thousand scoring records for 1000 users to evaluate 1700 films.

\subsection{Accuracy prediction}

The accuracy of prediction is considered to be the degree of similarity between the score of the recommended system prediction and the actual score of the user. In order to measure the accuracy of the recommendation, the accuracy of the recommended system is evaluated by means of the mean absolute error. In this formula, the smaller the mean absolute error, the higher the degree of similarity between the recommendation system and the user. The mean absolute error is as shown in formula (1).

$$
\mathrm{MAE}=\frac{1}{\mathrm{n}} \sum_{\alpha=1}^{n}\left(v_{i, \alpha}-v_{i, \alpha}\right)
$$

In the formula, $n$ is the number of movies that score the user $i$ in the system. $v_{i, a}$ is the prediction score of the recommended system. $r_{i, \alpha}$ is the actual score of the user $i$ for the movie. The accuracy calculated at the end is the average value of all users.

\subsection{Test methods}

Two methods are used to evaluate the performance of the system. The first method is to select a different set of neighbors from 5-25, to compare the traditional collaborative filtering algorithm and the MAE value of the improved algorithm. The second method is to observe the performance of the two algorithms when choosing different sparsity. The comparison of the traditional CF algorithm with the improved algorithm is shown in Figure 7. The RTE data model is shown in Figure 8. 


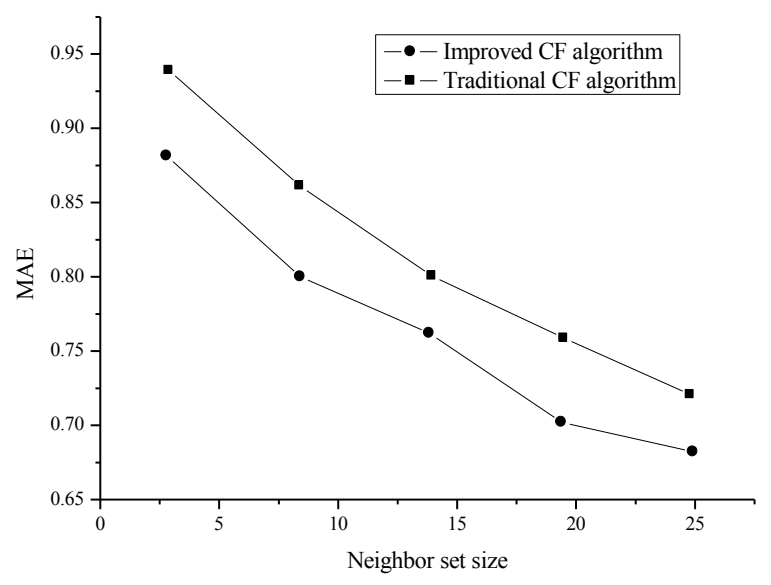

Fig. 7. The comparison of the traditional CF algorithm with the improved algorithm

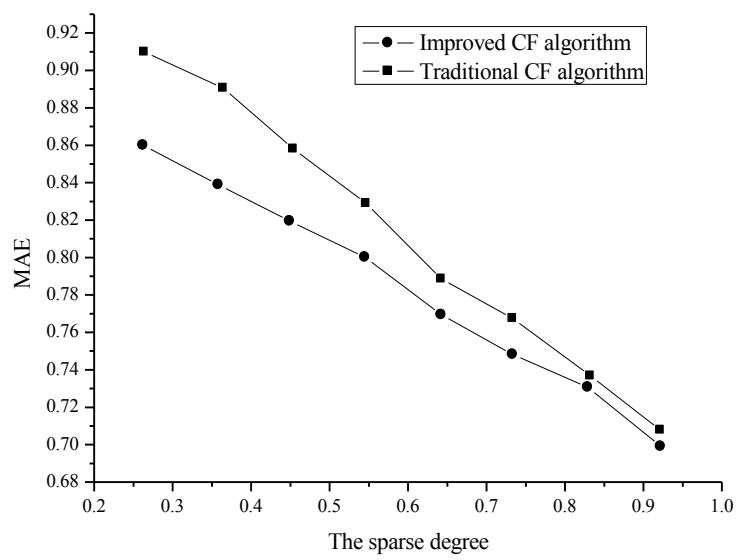

Fig. 8. The RTE data model

As can be seen from Figure 7, when the neighbor set is increased from 5 to 25, the improved collaborative filtering algorithm generally has a smaller MAE value than the traditional algorithm. In Figure 8, eight sets of data with a 0.2 0.9 range of sparsity are selected. The improved collaborative filtering algorithm is used to calculate the user's behavior weight when the sparsity is small. In a smaller sparsity environment, the prediction score is more accurate than that of the traditional algorithm.

Therefore, from the test results, the collaborative filtering recommendation algorithm is more accurate than the traditional algorithm. In the case of small sparsity, the recommendation effect is more obvious. 


\section{Conclusions}

The SCORM standard is very important. Collaborative filtering technology has been successfully used in the field of e-commerce. It has a positive impact on education. The traditional online training platform lacks effective resource integration and personalized resource recommendation. Collaborative filtering algorithm is introduced into the field of teaching resources. Combined with the SCORM standard, the design of the teaching resource library is optimized. The following conclusions are drawn.

1. An improved teaching resource management model is proposed. The model studies the combination of SCORM standards and personalized recommendation techniques.

2. The learning behavior state of the learners is tracked. An improved collaborative filtering model and algorithm is proposed, to alleviate the cold start and matrix sparsity in collaborative filtering algorithm.

3. Through the design of the model, the digital teaching resource base based on SCORM standard is realized. The repository can browse the teaching resources of the SCORM standard and learn the state of the learners.

4. The collaborative filtering algorithm can also provide the learners with their potential interest to achieve the effect of personalized recommendation. The learning efficiency of the learners is improved.

\section{Acknowledgement}

The National Information Technology Education Research of 2017 Youth Project(176140019).

\section{$7 \quad$ References}

[1] Rimale, Z., Benlahmar, E. H., \& Tragha, A. A New Responsive SCORM Design (RSCOD) Approach Based Semantic Learning Object (SLO) Using SharePoint Learning Kit (SLK). The, International Conference, 2017, vol. 2, pp. 1-6. https://doi.org/10.1145/ 3090354.3090387

[2] Silva, P. J. E. D., Oliveira, T. D., \& Pantoni, R. P. Integração de objetos de aprendizagem em matemática utilizando scorm em ambiente virtual de aprendizagem, 2017, vol. 110, pp 20.

[3] Zhu, X. H., Wu, T. J., \& Chen, H. C. An interoperable model for the intelligent content object based on a knowledge ontology and the scorm specification. Journal of Educational Computing Research, 2017, vol. 4, pp. 2576.

[4] Seixas, D. Scorm y la transformación digital o por qué debemos abandonar scorm. Observatorio De Recursos Humanos Y Relaciones Laborales, págs, 2017, vol. 5, pp. 56-58.

[5] Herbert, C., Velan, G. M., Pryor, W. M., \& Kumar, R. K. A model for the use of blended learning in large group teaching sessions. Bmc Medical Education, 2017, vol. 17(1), pp. 197. https://doi.org/10.1186/s12909-017-1057-2 
[6] Neves, D. E., Ishitani, L., \& Brandão, W. C. Metodologia para recomendação e agregação de objetos de aprendizagem no padrão scorm., 2016, vol. 24(1), pp. 11.

[7] Ruano, I., Cano, P., Gámez, J., \& Gómez, J. Advanced lms integration of scorm web laboratories. IEEE Access, 2016, vol. 4, pp. 6352-6363. https://doi.org/10.1109/ACCESS. 2016.2587805

[8] Lindert, Lisa|Su, Bude. The evolution of scorm to tin can api: implications for instructional design. Educational Technology, 2016, vol. (17), pp. 56.

[9] Ueda, H., \& Nakamura, M. Gakuninmoodle: toward robust e-learning services using moodle in japan ?. Procedia Computer Science, 2016, vol. (96), pp. 1710.

[10] Men, W., Wang, H., Cai, H., \& Centre, I. Research and design about the boutique resources sharing platform of colleges and universities in henan province. Journal of Xuchang University, 2017, vol. (82), pp. 171-178.

\section{Author}

Yan-Hong Shen is with Zhejiang Industry Polytechnic College, Zhejiang, China.

Article submitted 21 March 2018. Resubmitted 04 April 2018. Final acceptance 07 May 2018. Final version published as submitted by the author. 